\section{Global Spread of Multiple Aminoglycoside Resistance Genes}

\author{
Kunikazu Yamane, ${ }^{*}$ Jun-ichi Wachino,* \\ Yohei Doi, ${ }^{*}$ Hiroshi Kurokawa, ${ }^{*}$ \\ and Yoshichika Arakawa*
}

Emergence of the newly identified 16S rRNA methylases RmtA, RmtB, and ArmA in pathogenic gram-negative bacilli has been a growing concern. ArmA, which had been identified exclusively in Europe, was also found in several gram-negative pathogenic bacilli isolated in Japan, suggesting global dissemination of hazardous multiple aminoglycoside resistance genes.

$\mathrm{M}$ ultidrug-resistant gram-negative super microbes have been emerging worldwide. Since carbapenems and fluoroquinolones are the last resort against infections caused by gram-negative bacilli $(1,2)$, the proliferation and dissemination of such clinical isolates that produce metallo- $\beta$-lactamases and acquire mutations in gyrA and parC genes have become a global threat $(3,4)$. Aminoglycosides, including amikacin and tobramycin, are still potent agents for use against resistant bacilli. One of the most common resistance mechanisms against aminoglycosides is the production of aminoglycosidemodifying enzymes, such as aminoglycoside acetyltransferases, aminoglycoside phosphorylases, and aminoglycoside adenyltransferases (5), which are mainly mediated by transferable large plasmids.

Recently, a series of special methylases that protect microbial 16S rRNA, the main target of aminoglycosides, was identified in several nosocomial pathogens, including Pseudomonas aeruginosa (6), Serratia marcescens (7), and Klebsiella pneumoniae (8). The newly identified 16S rRNA methylases RmtA and RmtB were reported from Japan in 2003 and 2004, respectively (6,7). The gene for ArmA was initially sequenced in Citrobacter freundii isolated in Poland (GenBank accession no. AF550415) and later characterized in K. pneumoniae isolated in France in 2003 (8). In 2004, nosocomial spread of ArmA- or RmtBproducing Escherichia coli and K. pneumoniae was reported from Taiwan (9).

These enzymes are capable of conferring an extraordinary high level of resistance (MIC $>512 \mathrm{mg} / \mathrm{L}$ ) against most clinically important aminoglycosides as was

*National Institute of Infectious Diseases, Tokyo, Japan observed among aminoglycoside-producing actinomycetes, suggesting their probable phylogenic relationship with the intrinsic 16S rRNA methylases of actinomycetes (Figure). RmtA shared 82\% amino acid identity with $\mathrm{RmtB}$, but the amino acid sequence similarities between 16S rRNA methylases isolated from pathogenic gram-negative microbes and those from aminoglycoside-producing actinomycetes were relatively low ( $\leq 33 \%)$. From analyses of the genetic environments of genes encoding 16S rRNA methylases, the $r m t A$ gene is likely associated with the mercury-resistant transposon Tn5041 (10); the $r m t B$ gene was found in the flanking region of Tn3-like structure (7). The $\operatorname{arm} A$ gene was found on a large plasmid which carries a type 1 integron (8) that mediates various gene cassettes responsible for multiple antimicrobial resistance. The structure of these genetic environments implied that the genes for these 16S rRNA methylases are mediated by mobile genetic elements carried by transferable large plasmids $(7,8,10)$. In fact, the $r m t A$ gene was transferred from $P$. aeruginosa strain AR-2 to an aminoglycoside-susceptible $P$. aeruginosa strain 105 by conjugation in vitro (6). The $r m t B$ gene was also transferred from $S$. marcescens $S-$ 95 to $E$. coli by transformation (7). The armA gene was located on a composite transposon $\operatorname{Tn} 1548$ (11).

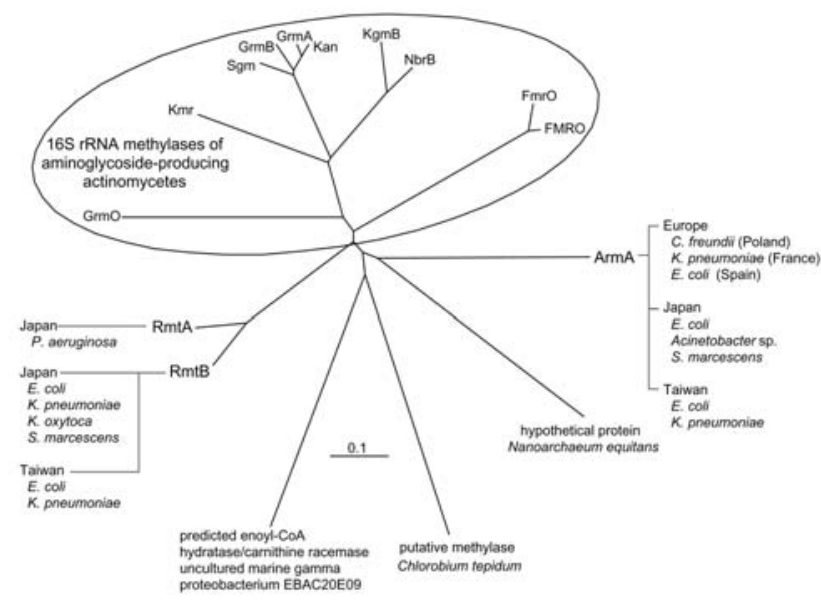

Figure. Phylogenic relationship among the 16S rRNA methylases. Each amino acid sequence was subjected to the analysis referred to the following sources: FmrO, accession no. JN0651; Kmr, accession no. AB164642; GrmA, accession no. M55520; GrmB, accession no. M55521; GrmO, accession no. AY524043; Kan, accession no. AJ414669; Sgm, accession no. A45282; KgmB, accession no. S60108; NbrB, accession no. AF038408; FMRO, Q08325; RmtA, (6); RmtB, (7); ArmA, (8); predicted enoyl-CoA hydratase/carnithine racemase of uncultured marine gamma proteobacterium EBAC20E09, accession no. AAS73112; putative methylase of Chlorobium tepidum, accession no. AAM72273; hypothetical protein of Nanoarchaeum equitans, accession no. AAR39385. The ClustalW program provided by the DNA Data Bank of Japan (http://www.ddbj.nig.ac.jp/search/clustalw-e.html) was used in this study. 
Thus, the growing concern was that these newly identified aminoglycoside-resistance genes could easily spread and be further disseminated among the glucose-nonfermentative gram-negative bacilli, including $P$. aeruginosa and Acinetobacter spp. and the genera belonging to the family Enterobacteriaceae.

\section{The Study}

We conducted a preliminary screening of the 16S rRNA methylase-producing bacilli on our gram-negative microbial stock of 2,877 strains isolated from Japanese hospitals within the past several years. Arbekacin, a semisynthetic aminoglycoside belonging to the kanamycin group, requires 2 modifications at the $\left(6^{\prime}\right)$ aminogroup and the $\left(2^{\prime \prime}\right)$ hydroxyl group for inactivation, so this agent is not inactivated by known plasmid-mediated aminoglycosidemodifying enzymes. Therefore, a high-level arbekacin resistance (MIC $>512 \mathrm{mg} / \mathrm{L}$ ) was used as a marker for screening the 16S rRNA methylase-producing strains. All arbekacin-resistant strains were subjected to polymerase chain reaction (PCR) analysis to detect $r m t A, r m t B$, or $\operatorname{arm} A$, and all strains were PCR positive, except for a strain of Acinetobacter demonstrating a very high level of resistance to arbekacin (MIC 1,024 mg/L). This strain was later shown to produce both aminoglycoside 6 '-acetyltransferase and 2 "-adenyltransferase (12), so arbekacin was inactivated in this strain by both 6'-acetylation and 2"adenylation. Each PCR primer set was used to detect $r m t A$ and $r m t B$ genes as in our previous reports $(6,7)$. The PCR primers for amplification of $\operatorname{arm} A$ were newly designed (forward: 5'-AGG TTG TTT CCA TTT CTG AG-3', reverse: $5^{\prime}$-TCT CTT CCA TTC CCT TCT CC-3'), and the predicted size of the amplicon was $590 \mathrm{bp}$. These 3 sets of PCR primers were very reliable in detecting $r m t A, r m t B$, and $\operatorname{arm} A$ genes, respectively. Each PCR amplicon was then subjected to sequencing analyses on both strands to confirm its nucleotide sequences for detecting mutations in the methylase genes.

As reported in our previous study, $r m t A$ and $r m t B$ genes had been found in $P$. aeruginosa isolates $(6,10)$ and in 1 strain of $S$. marcescens (7), respectively. As shown in the Table, 5 P. aeruginosa strains isolated after our previous report (6) were $r m t A$ positive. The $r m t B$ gene was additionally identified in $4 \mathrm{~K}$. pneumoniae, $2 \mathrm{E}$. coli, and $1 \mathrm{~K}$. oxytoca strains in Japan. To our surprise, the armA gene, which had been found in various gram-negative microbial species belonging to the family Enterobacteriaceae exclusively in Europe as reported by Galimand et al. (13), was also identified in Japan in 1 strain each of E. coli, S. marcescens, and Acinetobacter sp. Notably, the $\operatorname{arm} A$ and $r m t B$ genes were also recently identified in $K$. pneumoniae and E. coli in Taiwan (9). Furthermore, the genetic environment of the armA gene found in $C$. freundii isolated in Poland was similar to that of $K$. pneumoniae isolated in France. The genetic environments of the armA gene found in the 3 Japanese microbial species, E. coli, S. marcescens, and Acinetobacter sp. (GenBank accession nos. AB116388 and AB117519), were also similar to those found in Europe (GenBank accession nos. AF550415 and AY220558). These findings suggest that the ArmA-producing gram-negative nosocomial microbes that harbor a very similar genetic environment carrying the $\operatorname{arm} A$ gene have spread globally.

\section{Conclusions}

As described previously, arbekacin still shows a very broad antimicrobial spectrum from gram-positive to gramnegative nosocomial microbes and has been approved solely to treat methicillin-resistant Staphylococcus aureus (MRSA) infections in Japan since 1990 to ensure the prudent use of this agent. The emergence and presence of the 16S rRNA methylase-producing gram-negative bacilli,

\begin{tabular}{|c|c|c|c|c|}
\hline Species and strain & Type & Year of isolation & Hospital & Prefecture \\
\hline Pseudomonas aeruginosa $\mathrm{P} 122$ & $\mathrm{Rmt} A$ & 2002 & A & Aichi \\
\hline$P$. aeruginosa $\mathrm{P} 340$ & $\mathrm{RmtA}$ & 2002 & B & Gifu \\
\hline$P$. aeruginosa $02-386$ & $\mathrm{Rmt} A$ & 2002 & C & Saitama \\
\hline$P$. aeruginosa $03-29$ & $\mathrm{Rmt} A$ & 2003 & D & Aichi \\
\hline$P$. aeruginosa $03-230$ & RmtA & 2003 & $E$ & Shizuoka \\
\hline Escherichia coli 01-139 & RmtB & 2001 & $\mathrm{H}$ & Yamanashi \\
\hline Klebsiella pneumoniae 01-140 & RmtB & 2001 & $\mathrm{H}$ & Yamanashi \\
\hline Klebsiella oxytoca 01-141 & RmtB & 2001 & $\mathrm{H}$ & Yamanashi \\
\hline K. pneumoniae 01-142 & RmtB & 2001 & $\mathrm{H}$ & Yamanashi \\
\hline E. coli C316 & RmtB & 2002 & $\mathrm{~F}$ & Hyogo \\
\hline Serratia marcescens S95 & RmtB & 2002 & G & Kohchi \\
\hline K. pneumoniae 03-252 & RmtB & 2003 & $\mathrm{H}$ & Yamanashi \\
\hline K. pneumoniae 03-518 & RmtB & 2003 & $\mathrm{H}$ & Yamanashi \\
\hline E. coli C316-2 & ArmA & 2003 & $\mathrm{~F}$ & Hyogo \\
\hline S. marcescens ARS 8 & ArmA & 2003 & I & Tochigi \\
\hline Acinetobacter sp. ARS6 & ArmA & 2003 & J & Kanagawa \\
\hline
\end{tabular}


however, has not been well recognized in Japan to date; arbekacin has not been listed among the antimicrobial agents for daily antimicrobial susceptibility testing of gram-negative microbes.

The use of semisynthetic aminoglycosides, including arbekacin, in Japanese clinical settings for $>10$ years may have promoted the emergence and dissemination of the 16S rRNA methylase-producing gram-negative microbes in Japan. The large amount of various aminoglycosides used in livestock-farming environments could have also been a selective pressure for the emergence and spread of pathogenic microbes that harbor genetic determinants for the newly identified 16S rRNA methylases, as exemplified by recent isolation of ArmA-producing E. coli from swine in Spain (GenBank accession no. AY522431).

Since acquisition of multidrug resistance against clinically important antimicrobial agents such as carbapenems and fluoroquinolones has been developing rapidly worldwide, the acceleration of even greater aminoglycoside resistance among gram-negative bacilli promises to become an actual clinical concern in the near future, just as vancomycin-resistant enterococci (VRE) did in the 1990s (14). The emergence of gram-positive cocci including MRSA and VRE that acquire the 16S rRNA methylase could also be a grave clinical matter, although fortunately no such hazardous microbes have been identified. Thus, steps must be taken to block further proliferation of these multidrug-resistant gram-negative super microbes, including P. aeruginosa, K. pneumoniae, and Acinetobacter spp., as well as multidrug-resistant cocci such as MRSA and VRE, which have acquired an extraordinarily high level of resistance to various aminoglycosides through production of 16S rRNA methylases, especially in clinical environments.

This work was funded in part by grants from the Ministry of Health, Labor and Welfare, Japan (H15-Shinkou-9, H15Shinkou-10) and Grant-in-Aid for Young Scientists (B) 16790318 from the Ministry of Education, Culture, Sports, Science and Technology, Japan.

Dr. Yamane, a physician with background in intensive care and emergency medicine, is currently working at National Institute of Infectious Diseases, Japan. His current research interest is molecular mechanisms of antimicrobial resistance in nosocomial bacteria.

The opinions expressed by authors contributing to this journal do not necessarily reflect the opinions of the Centers for Disease Control and Prevention or the institutions with which the authors are affiliated.

\section{References}

1. Schwaber MJ, Cosgrove SE, Gold HS, Kaye KS, Carmeli Y. Fluoroquinolones protective against cephalosporin resistance in gram-negative nosocomial pathogens. Emerg Infect Dis. 2004;10:94-9.

2. Zanetti G, Bally F, Greub G, Garbino J, Kinge T, Lew D, et al. Cefepime versus imipenem-cilastatin for treatment of nosocomial pneumonia in intensive care unit patients: a multicenter, evaluatorblind, prospective, randomized study. Antimicrob Agents Chemother. 2003;47:3442-7.

3. Kurokawa H, Yagi T, Shibata N, Shibayama K, Arakawa Y. Worldwide proliferation of carbapenem-resistant gram-negative bacteria. Lancet. 1999;354:955.

4. Sahm DF, Thornsberry C, Jones ME, Karlowsky JA. Factors influencing fluoroquinolone resistance. Emerg Infect Dis. 2003;9:1651-4.

5. Shaw KJ, Rather PN, Hare RS, Miller GH. Molecular genetics of aminoglycoside resistance genes and familial relationships of the aminoglycoside-modifying enzymes. Microbiol Rev. 1993;57:138-63.

6. Yokoyama K, Doi Y, Yamane K, Kurokawa H, Shibata N, Shibayama K, et al. Acquisition of 16S rRNA methylase gene in Pseudomonas aeruginosa. Lancet. 2003;362:1888-93.

7. Doi Y, Yokoyama K, Yamane K, Wachino J, Shibata N, Yagi T, et al. Plasmid-mediated 16S rRNA methylase in Serratia marcescens conferring high-level resistance to aminoglycosides. Antimicrob Agents Chemother. 2004;48:491-6.

8. Galimand M, Courvalin P, Lambert T. Plasmid-mediated high-level resistance to aminoglycosides in Enterobacteriaceae due to $16 \mathrm{~S}$ rRNA methylation. Antimicrob Agents Chemother. 2003;47:2565-71.

9. Yan JJ, Wu JJ, Ko WC, Tsai SH, Chuang CL, Wu HM, et al. Plasmidmediated 16S rRNA methylases conferring high-level aminoglycoside resistance in Escherichia coli and Klebsiella pneumoniae isolates from two Taiwanese hospitals. J Antimicrob Chemother. 2004;54:1007-12.

10. Yamane K, Doi Y, Yokoyama K, Yagi T, Kurokawa H, Shibata N, et al. Genetic environments of the rmtA gene found in Pseudomonas aeruginosa clinical isolates. Antimicrob Agents Chemother. 2004;48:2069-74.

11. Lambert T, Galimand M, Sabtcheva S, Courvalin P. The armA aminoglycoside resistance methylase gene is borne by composite transposon Tn1548 [Abstract C1-1496]. Presented at the 44th Interscience Conference on Antimicrobial Agents and Chemotherapy; Washington; 2004 Oct 30-Nov 2.

12. Doi Y, Wachino J, Yamane K, Shibata N, Yagi T, Shibayama K, et al. Spread of novel aminoglycoside resistance gene aac(6')-Iad among Acinetobacter clinical isolates in Japan. Antimicrob Agents Chemother. 2004;48:2075-80.

13. Galimand M, Sabtcheva S, Kantardjiev T, Poirel L, Arlet G, Courvalin $\mathrm{P}$, et al. The $\operatorname{arm} A$ aminoglycoside resistance methylase gene is disseminated in Enterobacteriaceae by an incL/M plasmid mediating CTX-M-beta-lactamase [Abstract C2-59]. Presented at the 43rd Interscience Conference on Antimicrobial Agents and Chemotherapy; Chicago; 2003 Sep 14-17.

14. Frieden TR, Munsiff SS, Low DE, Willey BM, Williams G, Faur Y, et al. Emergence of vancomycin-resistant enterococci in New York City. Lancet. 1993;342:76-9.

Address for correspondence: Yoshichika Arakawa, Department of Bacterial Pathogenesis and Infection Control, National Institute of Infectious Diseases, 4-7-1 Gakuen, Musashi-Murayama, Tokyo 2080011, Japan; fax: 81-42-461-7173; email: yarakawa@nih.go.jp 